UT-ICEPP 95-01

March 1995

\title{
A search for massive neutral bosons in orthopositronium decay
}

\author{
T. Maenot, M. Fujikawa, J. Kataoka, Y. Nishihara, \\ S. Orito, K. Shigekuni and Y. Watanabe \\ Department of Physics, Faculty of Science, \\ University of Tokyo, Tokyo 113, Japan
}

\begin{abstract}
We have searched for an exotic decay of orthopositronium into a single photon and a short-lived neutral boson in the hitherto unexplored mass region above $900 \mathrm{keV} / c^{2}$, by noting that this decay is one of few remaining candidates which could explain the discrepancy of the orthopositronium decay-rate. A high-resolution measurement of the associated photon energy spectrum was carried out with a germanium detector to search for a sharp peak from this two-body decay. Our negative result provides the upper-limits of $2.0 \times 10^{-4}$ on the branching ratio of such a decay in the mass region from 847 to $1013 \mathrm{keV} / \mathrm{c}^{2}$, and excludes the possibility of this decay mode explaining the discrepancy in the orthopositronium decay-rate.
\end{abstract}

\section{ICEPP}

International Center for Elementary Particle Physics, Faculty of Science, University of Tokyo

7-3-1 Hongo, Bunkyo-ku, Tokyo 113, Japan

${ }^{*}$ Submitted for publication in Physics Letters B

${ }^{\dagger}$ E-mail address: maeno@uticeaix1.icepp.s.u-tokyo.ac.jp 
Over the last seven years, three precision measurements of the orthopositronium (o-Ps) decay-rate were performed, which report the decay-rate faster than the QED prediction [1] by 1400, 1300 and 920 ppm, respectively, corresponding to $6.7,6.3$ and 4.3 standard deviations [2]. In attempts to explain the origin of this discrepancy, various exotic $o-P$ s decay modes have been investigated without obtaining any evidence so far. The decay mode into invisible final states was ruled out at $3 \mathrm{ppm} \mathrm{[3].} \mathrm{The} \mathrm{decay} \mathrm{into} \mathrm{two} \mathrm{and} \mathrm{four} \mathrm{photons,} \mathrm{forbidden}$ by space-rotational invariance and by the charge-conjugation invariance of QED, was excluded at 233 and $8 \mathrm{ppm}$, respectively [4, 5]. The decay into two photons and a long-lived, weakly-interacting vector boson was excluded at $10 \mathrm{ppm}$ [6]. The decay into a photon and a long-lived, weakly-interacting particle was also ruled out at $10 \mathrm{ppm}$ for a mass less than $1010 \mathrm{keV} / \mathrm{c}^{2}$ []], while that into a photon and a short-lived neutral boson with a mass less than $900 \mathrm{keV} / c^{2}$ was excluded at $400 \mathrm{ppm}$ [8, 9, 10]. One of very few remaining candidates for the source of the discrepancy is the decay into a single photon and a short-lived neutral boson $X^{0}$ in the hitherto unexplored mass range above $900 \mathrm{keV} / c^{2}$. We report in this letter a dedicated search for such a decay.

A schematic view of the experimental setup is shown in Fig. 1. A ${ }^{22} \mathrm{Na}$ positron source of intensity $0.7 \mu \mathrm{Ci}$ ( $2 \mathrm{~mm}$ spot diameter) is sandwiched between a plastic scintillator (NE104) of thickness $100 \mu \mathrm{m}$ and a mylar sheet of thickness $25 \mu \mathrm{m}$, each with a diameter of $12 \mathrm{~mm}$. The source and a target of silica aerogel (dimensions $10 \times 10 \times 5 \mathrm{~mm}^{3}$, density $0.1 \mathrm{~g} \mathrm{~cm}^{-3}$ ) are held at the center of a pipe (18 mm diameter) made of $20 \mu \mathrm{m}$ thick aluminized mylar. The pipe is filled with $\mathrm{N}_{2}$ gas at 1 atmosphere. Most of the positrons emitted toward the target pass through the scintillator, giving light pulses to two photomultipliers (Hamamatsu $\mathrm{H}-3165-\mathrm{PV})$. When stopped in the silica aerogel, they form positronium-atoms for about $20 \%$ of cases. The total efficiency of $o-P$ s formation in our set-up was measured to be $3.0 \%$ per $\beta^{+}$decay.

The energy of the photon from the decay of $o-P s$ is measured by a planar high-purity germanium detector (diameter $16 \mathrm{~mm}$, thickness $10 \mathrm{~mm}$, Ortec GLP16195/10-P). The germanium detector has a thin Be window of thickness 0.13 $\mathrm{mm}$, which results to a high efficiency to low-energy photons down to $5 \mathrm{keV}$. The energy resolution and the absolute peak efficiencies of the germanium detector are determined as a function of the photon-energy by using the line $\gamma$ peaks from various sources of known strength, i.e., ${ }^{22} \mathrm{Na},{ }^{57} \mathrm{Co},{ }^{133} \mathrm{Ba},{ }^{152} \mathrm{Eu},{ }^{210} \mathrm{~Pb}$ and ${ }^{226} \mathrm{Ra}$, placed at the source position. The energy resolution obtained is 270, 525 and $828 \mathrm{eV}$ FWHM at $14.4,136.5$ and $356.0 \mathrm{keV}$, respectively.

The trigger and the data-acquisition system is arranged as follows. The pulses from the two photomultipliers are discriminated individually and the coincidences between them provide the start signals to the time-to-digital converter (TDC ; LeCroy 2228A), as well as the trigger signals to the CAMAC system. One output from the preamplifier of the germanium detector is fed through a fast-filter 
amplifier (Ortec 474) with an integration-time of 100 nsec, into the peak-hold analog-to-digital converter (ADC-1 ; Hohshin C008), and also into a discriminator whose output is used as the stop signal for the TDC. The other preamplifier output is amplified by a shaping amplifier (Ortec 572) with an integration-time of $3 \mu \mathrm{sec}$. One output of the shaping amplifier is fed into the peak-hold analogto-digital converter (ADC-2 ; Hohshin C011), to record the information of the low-energy region below $160 \mathrm{keV}$. The other output of the shaping amplifier is attenuated and fed into the other input of the ADC-2, to record the energy information in a wider range up to $600 \mathrm{keV}$. If the TDC stop signal is not generated within $5 \mu$ sec after the trigger, the data-readout is terminated and the system is cleared to accept the next trigger.

The data were collected from 10 runs for a total of $2.26 \times 10^{6} \mathrm{sec}$. Throughout the data-taking period, the room temperature was controlled to $\pm 0.4^{\circ} \mathrm{C}$. The energy calibrations and the measurements of the energy resolution were carried out at intervals of 7 days. In addition, the position and the width of the $511 \mathrm{keV}$ annihilation line were monitored by offline analysis of the data, which showed a stable peak position within $0.03 \mathrm{keV}$ and a constant energy-resolution throughout the data-taking period.

The offline selection and calibration process is as follows. In order to eliminate the pile-up effects, we demand the consistency between two ADC values with different integration-time : If the ratio of the two values deviates from the central value by more than $6 \%$, the event is rejected as being affected by the pile-up. This selection has an efficiency of $92 \%$. The time walk of the discriminator output is corrected by using the pulse height information from the ADC-2. The nonlinearlity of the TDC is corrected by using a flat spectrum between random triggers and clock signals.

Figure 2 shows the time spectrum between the scintillator and germanium signals. A sharp peak of the prompt annihilations is followed by the exponential decay of $o-$ Ps and subsequently by the constant accidentals. The time spectrum fits well to an exponential function plus a constant term. A decay time of 134.2 $\pm 0.4 \mathrm{nsec}$ is consistently obtained for various time spans if the fitting starts more than 150 nsec after the prompt peak. To obtain a pure sample of the $o-\mathrm{Ps}$ decay, we select the events in the time-window between 150 and 400 nsec after the prompt annihilations. The accidental contribution is measured by using the events in the time-window between 2000 and 3200 nsec after the prompt peak.

Figures $3 \mathrm{a}$ and $3 \mathrm{~b}$ show the measured energy-spectra from the $o-P$ s decay ( $o-$ Ps spectrum), thus selected and the accidental contribution being subtracted. The two-body decay into a photon and $X^{0}\left(\gamma X^{0}\right.$ decay) would appear in this $o-$ Ps spectrum as a narrow peak on a smooth background. The peak position $k_{p}$ is related to the $X^{0}$ mass, $m_{X^{0}}$, by

$$
k_{p}=m_{e}\left[1-\left(m_{X^{0}} / 2 m_{e}\right)^{2}\right] \text {, }
$$


where $m_{e}$ is the electron mass.

Peaks in the $o-$ Ps spectrum have been searched for by scanning $k_{p}$ with a step of $0.088 \mathrm{keV}$ from 10 to $160 \mathrm{keV}$, corresponding to the $m_{X^{0}}$ region from 847 to $1013 \mathrm{keV} / c^{2}$ : At each $k_{p}$, the $o-$ Ps spectrum was fitted with the function

$$
S(k)+\mathbf{n}\left(k_{p}\right) P(k),
$$

where $S(k)$ and $P(k)$ represent the smooth background-spectrum and the normalized peak-function, respectively. The fitting parameter $\mathbf{n}\left(k_{p}\right)$ corresponds to the number of photons from the $\gamma X^{0}$ decay events under the peak at $k_{p}$. The background-spectrum $S(k)$ is obtained at each $k_{p}$ by fitting the $o$-Ps spectrum (excluding the region within 2.5 times the FWHM of the peak position) with a polynomial function of up to seventh order. Since the intrinsic width of $X^{0}$ is expected to be extremely small, the observable width of the peak would be dominated by the energy-resolution of the detector and by the thermal motion of $o-\mathrm{Ps}$. The thermal kinetic energy of $o-\mathrm{Ps}_{\mathrm{s}}$ has been measured in an aerogel similar to the one used in this experiment and is known to decrease to about 0.04 $\mathrm{eV}$ within $60 \mathrm{nsec}$ after formation [11, 12]. The Doppler-broadening due to this thermal kinetic energy would contribute negligibly to the peak-width. Therefore the shape of the peak is assumed to be Gaussian, of a width determined by the energy-resolution of the germanium detector.

No statistically significant peak beyond 4 standard deviations was detected in the $o-$ Ps spectrum, resulting to upper limits on $\mathbf{n}\left(k_{p}\right)$. The limits on $\mathbf{n}\left(k_{p}\right)$ can be converted into the corresponding limits on the branching ratio of the $\gamma X^{0}$ decay, $\operatorname{Br}\left(k_{p}\right)$, by using the formula

$$
\operatorname{Br}\left(k_{p}\right)=\frac{\mathbf{n}\left(k_{p}\right)}{\epsilon\left(k_{p}\right) N_{\text {ops }}}
$$

where $\epsilon\left(k_{p}\right)$ is the absolute peak efficiency for a $\gamma$-ray of energy $k_{p}$.

$N_{\text {ops }}$ represents the total number of the $o-$ Ps decays occurring within the 150 - 400 nsec time-window, and is determined by fitting the "compton-free" region of the $o-P s$ spectrum (between 400 to $490 \mathrm{keV}$, where the three-photon decays dominate) with the theoretical spectrum [13] of the three-photon decay folded with the energy-dependent efficiency. It should be noted that $\operatorname{Br}\left(k_{p}\right)$ thus obtained has a small systematic error, being dependent only on the relative values of the efficiencies, not on their absolute values.

The resulting upper-limits at a $90 \%$ confidence level on the branching ratio of $\gamma X^{0}$ decay are shown in Fig. 4 , as a function of $m_{X^{0}}$. As indicated in this figure, new upper limits on the $\gamma X^{0}$ branching ratio in the mass range of $X^{0}$ from 900 to $1013 \mathrm{keV} / c^{2}$ have been obtained. Since our upper limits are at least a factor of 7 below the level which would explain the reported discrepancy of the $o-\mathrm{Ps}$ lifetime, we conclude that the $o-$ Ps decay mode of $\gamma X^{0}$ in the mass region below 
$1013 \mathrm{keV} / c^{2}$ can not explain the reported discrepancy of the orthopositronium decay-rate.

We wish to thank Dr. S. Asai for suggestions and discussions, Dr. X. Zhang for kindly lending us the germanium detector, Mr. Y. Nakamura, Mr. N. Kimura and Mr. M. Matsui, of Japan Radioisotope Association, for preparing the positron source. The analysis was performed on the RS/6000 workstations supplied for partnership program between ICEPP and IBM Japan Ltd. 


\section{References}

[1] W. E. Caswell, G. P. Lepage and J. Sapirstein, Phys. Rev. Lett. 38 (1977) 488; G. S. Adkins, Ann. Phys. (N.Y.) 146 (1983) 78; G. S. Adkins, A. A. Salahuddin and K. E. Schalm, Phys. Rev. A 45 (1992) 3333; 45 (1992) 7774.

[2] C. I. Westbrook, D. W. Gidley, R. S. Conti and A. Rich, Phys. Rev. Lett. 58 (1987) 1328; Phys. Rev. A 40 (1989) 5489; J. S. Nico, D. W. Gidley, A. Rich and P. W. Zitzewitz, Phys. Rev. Lett. 65 (1990) 1344.

[3] T. Mitsui, R. Fujimoto, Y. Ishisaki, Y .Ueda, Y. Yamazaki, S. Asai and S. Orito, Phys. Rev. Lett. 70 (1993) 2265; G. S. Atoyan, S. N. Gninenko, V. I. Razin and Y. V. Ryabov, Phys. Lett. B 220 (1989) 317.

[4] S. Asai, S. Orito, T. Sanuki, M. Yasuda and T. Yokoi, Phys. Rev. Lett. 66 (1991) 1298; D. W. Gidley, J. S. Nico and M. Skalsey, Phys. Rev. Lett. 66 (1991) 1302.

[5] K. Marko and A. Rich, Phys. Rev. Lett. 33 (1974) 980.

[6] T. Mitsui, K. Maki, N. Muramoto, T. Sato and S. Asai, in preparation.

[7] S. Asai, S. Orito, K. Yoshimura and T. Haga, Phys. Rev. Lett. 66 (1991) 2440; S. Orito, K. Yoshimura, T. Haga and M. Tsuchiaki, Phys. Rev. Lett. 63 (1989) 597.

[8] M. Tsuchiaki, S. Orito, T. Yoshida and M. Minowa, Phys. Lett. B 236 (1990) 81.

[9] M. V. Akopyan, G. S. Atoyan, S. N. Gninenko and V. V. Sukhov, Phys. Lett. B 272 (1991) 443.

[10] S. Asai, K. Shigekuni, T. Sanuki and S. Orito, Phys. Lett. B 323 (1994) 90.

[11] T. Chang, M. Xu, and X. Zeng, Phys. Lett. A 126 (1987) 189.

[12] T. Hyodo et al., in positron Annihilation, edited by L. Dorikens-Vanbraet, M. Dorikens and D. Segers (World Scientific, Singapore, 1989), p. 878.

[13] G. S. Adkins, Ann.Phys. (N.Y.) 146 (1983) 78. 


\section{Figure captions}

Fig. 1. A schematic view of the experimental setup. Circular inset: a magnified view of the source region.

Fig. 2. The time spectrum between the scintillator and germanium detector signals.

Fig. 3. The $o-$ Ps spectra after subtracting the accidental contribution. (a) Below $160 \mathrm{keV}$. (b) Up to $600 \mathrm{keV}$.

Fig. 4. The resultant upper-limits at $90 \%$ C.L. on the branching ratio of $\gamma X^{0}$ decay in comparison with the existing limits [8]. 
This figure "fig1-1.png" is available in "png" format from: http://arxiv.org/ps/hep-ex/9503004v1 
This figure "fig1-2.png" is available in "png" format from: http://arxiv.org/ps/hep-ex/9503004v1 
This figure "fig1-3.png" is available in "png" format from: http://arxiv.org/ps/hep-ex/9503004v1 
This figure "fig1-4.png" is available in "png" format from: http://arxiv.org/ps/hep-ex/9503004v1 
UT-ICEPP 95-01

March 1995

\title{
A search for massive neutral bosons in orthopositronium decay*
}

\author{
T. Maeno ${ }^{\dagger}$, M. Fujikawa, J. Kataoka, Y. Nishihara, \\ S. Orito, K. Shigekuni and Y. Watanabe \\ Department of Physics, Faculty of Science, \\ University of Tokyo, Tokyo 113, Japan
}

\begin{abstract}
We have searched for an exotic decay of orthopositronium into a single photon and a short-lived neutral boson in the hitherto unexplored mass region above $900 \mathrm{keV} / c^{2}$, by noting that this decay is one of few remaining candidates which could explain the discrepancy of the orthopositronium decay-rate. A high-resolution measurement of the associated photon energy spectrum was carried out with a germanium detector to search for a sharp peak from this two-body decay. Our negative result provides the upper-limits of $2.0 \times 10^{-4}$ on the branching ratio of such a decay in the mass region from 847 to $1013 \mathrm{keV} / c^{2}$, and excludes the possibility of this decay mode explaining the discrepancy in the orthopositronium decay-rate.
\end{abstract}

\section{ICEPP}

International Center for Elementary Particle Physics,

Faculty of Science, University of Tokyo

7-3-1 Hongo, Bunkyo-ku, Tokyo 113, Japan

* Submitted for publication in Physics Letters B

${ }^{\dagger}$ E-mail address: maeno@uticeaix1.icepp.s.u-tokyo.ac.jp 
Over the last seven years, three precision measurements of the orthopositronium (o-Ps) decay-rate were performed, which report the decay-rate faster than the QED prediction [1] by 1400,1300 and $920 \mathrm{ppm}$, respectively, corresponding to $6.7,6.3$ and 4.3 standard deviations [2]. In attempts to explain the origin of this discrepancy, various exotic $o-P$ s decay modes have been investigated without obtaining any evidence so far. The decay mode into invisible final states was ruled out at $3 \mathrm{ppm}$ [3]. The decay into two and four photons, forbidden by space-rotational invariance and by the charge-conjugation invariance of QED, was excluded at 233 and $8 \mathrm{ppm}$, respectively [4,5]. The decay into two photons and a long-lived, weakly-interacting vector boson was excluded at $10 \mathrm{ppm}$ [6]. The decay into a photon and a long-lived, weakly-interacting particle was also ruled out at $10 \mathrm{ppm}$ for a mass less than $1010 \mathrm{keV} / c^{2}$ [7], while that into a photon and a short-lived neutral boson with a mass less than $900 \mathrm{keV} / c^{2}$ was excluded at $400 \mathrm{ppm}[8,9,10]$. One of very few remaining candidates for the source of the discrepancy is the decay into a single photon and a short-lived neutral boson $X^{0}$ in the hitherto unexplored mass range above $900 \mathrm{keV} / c^{2}$. We report in this letter a dedicated search for such a decay.

A schematic view of the experimental setup is shown in Fig. 1. A $^{22} \mathrm{Na}$ positron source of intensity $0.7 \mu \mathrm{Ci}(2 \mathrm{~mm}$ spot diameter) is sandwiched between a plastic scintillator (NE104) of thickness $100 \mu \mathrm{m}$ and a mylar sheet of thickness $25 \mu \mathrm{m}$, each with a diameter of $12 \mathrm{~mm}$. The source and a target of silica aerogel (dimensions $10 \times 10 \times 5 \mathrm{~mm}^{3}$, density $0.1 \mathrm{~g} \mathrm{~cm}^{-3}$ ) are held at the center of a pipe (18 mm diameter) made of $20 \mu \mathrm{m}$ thick aluminized mylar. The pipe is filled with $\mathrm{N}_{2}$ gas at 1 atmosphere. Most of the positrons emitted toward the target pass through the scintillator, giving light pulses to two photomultipliers (Hamamatsu $\mathrm{H}-3165-\mathrm{PV})$. When stopped in the silica aerogel, they form positronium-atoms for about $20 \%$ of cases. The total efficiency of $o-P$ s formation in our set-up was measured to be $3.0 \%$ per $\beta^{+}$decay.

The energy of the photon from the decay of $o-P s$ is measured by a planar high-purity germanium detector (diameter $16 \mathrm{~mm}$, thickness $10 \mathrm{~mm}$, Ortec GLP16195/10-P). The germanium detector has a thin Be window of thickness 0.13 $\mathrm{mm}$, which results to a high efficiency to low-energy photons down to $5 \mathrm{keV}$. The energy resolution and the absolute peak efficiencies of the germanium detector are determined as a function of the photon-energy by using the line $\gamma$ peaks from various sources of known strength, i.e., ${ }^{22} \mathrm{Na},{ }^{57} \mathrm{Co},{ }^{133} \mathrm{Ba},{ }^{152} \mathrm{Eu},{ }^{210} \mathrm{~Pb}$ and ${ }^{226} \mathrm{Ra}$, placed at the source position. The energy resolution obtained is 270,525 and $828 \mathrm{eV} \mathrm{FWHM}$ at $14.4,136.5$ and $356.0 \mathrm{keV}$, respectively.

The trigger and the data-acquisition system is arranged as follows. The pulses from the two photomultipliers are discriminated individually and the coincidences between them provide the start signals to the time-to-digital converter (TDC ; LeCroy $2228 \mathrm{~A}$ ), as well as the trigger signals to the CAMAC system. One output from the preamplifier of the germanium detector is fed through a fast-filter 
amplifier (Ortec 474) with an integration-time of $100 \mathrm{nsec}$, into the peak-hold analog-to-digital converter (ADC-1 ; Hohshin C008), and also into a discriminator whose output is used as the stop signal for the TDC. The other preamplifier output is amplified by a shaping amplifier (Ortec 572) with an integration-time of $3 \mu \mathrm{sec}$. One output of the shaping amplifier is fed into the peak-hold analogto-digital converter (ADC-2 ; Hohshin C011), to record the information of the low-energy region below $160 \mathrm{keV}$. The other output of the shaping amplifier is attenuated and fed into the other input of the $\mathrm{ADC}-2$, to record the energy information in a wider range up to $600 \mathrm{keV}$. If the TDC stop signal is not generated within $5 \mu$ sec after the trigger, the data-readout is terminated and the system is cleared to accept the next trigger.

The data were collected from 10 runs for a total of $2.26 \times 10^{6} \mathrm{sec}$. Throughout the data-taking period, the room temperature was controlled to $\pm 0.4^{\circ} \mathrm{C}$. The energy calibrations and the measurements of the energy resolution were carried out at intervals of 7 days. In addition, the position and the width of the $511 \mathrm{keV}$ annihilation line were monitored by offline analysis of the data, which showed a stable peak position within $0.03 \mathrm{keV}$ and a constant energy-resolution throughout the data-taking period.

The offline selection and calibration process is as follows. In order to eliminate the pile-up effects, we demand the consistency between two ADC values with different integration-time : If the ratio of the two values deviates from the central value by more than $6 \%$, the event is rejected as being affected by the pile-up. This selection has an efficiency of $92 \%$. The time walk of the discriminator output is corrected by using the pulse height information from the ADC-2. The nonlinearlity of the TDC is corrected by using a flat spectrum between random triggers and clock signals.

Figure 2 shows the time spectrum between the scintillator and germanium signals. A sharp peak of the prompt annihilations is followed by the exponential decay of $o-P$ s and subsequently by the constant accidentals. The time spectrum fits well to an exponential function plus a constant term. A decay time of 134.2 $\pm 0.4 \mathrm{nsec}$ is consistently obtained for various time spans if the fitting starts more than 150 nsec after the prompt peak. To obtain a pure sample of the $o-P s$ decay, we select the events in the time-window between 150 and $400 \mathrm{nsec}$ after the prompt annihilations. The accidental contribution is measured by using the events in the time-window between 2000 and 3200 nsec after the prompt peak.

Figures $3 \mathrm{a}$ and $3 \mathrm{~b}$ show the measured energy-spectra from the $o-P$ s decay (o-Ps spectrum), thus selected and the accidental contribution being subtracted. The two-body decay into a photon and $X^{0}\left(\gamma X^{0}\right.$ decay) would appear in this $o-$ Ps spectrum as a narrow peak on a smooth background. The peak position $k_{p}$ is related to the $X^{0}$ mass, $m_{X^{0}}$, by

$$
k_{p}=m_{e}\left[1-\left(m_{X^{0}} / 2 m_{e}\right)^{2}\right],
$$


where $m_{e}$ is the electron mass.

Peaks in the $o-P$ s spectrum have been searched for by scanning $k_{p}$ with a step of $0.088 \mathrm{keV}$ from 10 to $160 \mathrm{keV}$, corresponding to the $m_{X^{0}}$ region from 847 to $1013 \mathrm{keV} / c^{2}$ : At each $k_{p}$, the $o-$ Ps spectrum was fitted with the function

$$
S(k)+\mathbf{n}\left(k_{p}\right) P(k),
$$

where $S(k)$ and $P(k)$ represent the smooth background-spectrum and the normalized peak-function, respectively. The fitting parameter $\mathbf{n}\left(k_{p}\right)$ corresponds to the number of photons from the $\gamma X^{0}$ decay events under the peak at $k_{p}$. The background-spectrum $S(k)$ is obtained at each $k_{p}$ by fitting the $o$-Ps spectrum (excluding the region within 2.5 times the FWHM of the peak position) with a polynomial function of up to seventh order. Since the intrinsic width of $X^{0}$ is expected to be extremely small, the observable width of the peak would be dominated by the energy-resolution of the detector and by the thermal motion of $o-P$ s. The thermal kinetic energy of $o-P s$ has been measured in an aerogel similar to the one used in this experiment and is known to decrease to about 0.04 $\mathrm{eV}$ within $60 \mathrm{nsec}$ after formation $[11,12]$. The Doppler-broadening due to this thermal kinetic energy would contribute negligibly to the peak-width. Therefore the shape of the peak is assumed to be Gaussian, of a width determined by the energy-resolution of the germanium detector.

No statistically significant peak beyond 4 standard deviations was detected in the $o-P$ s spectrum, resulting to upper limits on $\mathbf{n}\left(k_{p}\right)$. The limits on $\mathbf{n}\left(k_{p}\right)$ can be converted into the corresponding limits on the branching ratio of the $\gamma X^{0}$ decay, $B r\left(k_{p}\right)$, by using the formula

$$
B r\left(k_{p}\right)=\frac{\mathbf{n}\left(k_{p}\right)}{\epsilon\left(k_{p}\right) N_{o p s}},
$$

where $\epsilon\left(k_{p}\right)$ is the absolute peak efficiency for a $\gamma$-ray of energy $k_{p}$.

$N_{\text {ops }}$ represents the total number of the $o-P$ s decays occurring within the 150 - 400 nsec time-window, and is determined by fitting the "compton-free" region of the $o-P$ s spectrum (between 400 to $490 \mathrm{keV}$, where the three-photon decays dominate) with the theoretical spectrum [13] of the three-photon decay folded with the energy-dependent efficiency. It should be noted that $\operatorname{Br}\left(k_{p}\right)$ thus obtained has a small systematic error, being dependent only on the relative values of the efficiencies, not on their absolute values.

The resulting upper-limits at a $90 \%$ confidence level on the branching ratio of $\gamma X^{0}$ decay are shown in Fig. 4 , as a function of $m_{X^{0}}$. As indicated in this figure, new upper limits on the $\gamma X^{0}$ branching ratio in the mass range of $X^{0}$ from 900 to $1013 \mathrm{keV} / \mathrm{c}^{2}$ have been obtained. Since our upper limits are at least a factor of 7 below the level which would explain the reported discrepancy of the $o-P s$ lifetime, we conclude that the $o-P$ s decay mode of $\gamma X^{0}$ in the mass region below 
$1013 \mathrm{keV} / \mathrm{c}^{2}$ can not explain the reported discrepancy of the orthopositronium decay-rate.

We wish to thank Dr. S. Asai for suggestions and discussions, Dr. X. Zhang for kindly lending us the germanium detector, Mr. Y. Nakamura, Mr. N. Kimura and Mr. M. Matsui, of Japan Radioisotope Association, for preparing the positron source. The analysis was performed on the RS/6000 workstations supplied for partnership program between ICEPP and IBM Japan Ltd. 


\section{References}

[1] W. E. Caswell, G. P. Lepage and J. Sapirstein, Phys. Rev. Lett. 38 (1977) 488; G. S. Adkins, Ann. Phys. (N.Y.) 146 (1983) 78; G. S. Adkins, A. A. Salahuddin and K. E. Schalm, Phys. Rev. A 45 (1992) 3333; 45 (1992) 7774.

[2] C. I. Westbrook, D. W. Gidley, R. S. Conti and A. Rich, Phys. Rev. Lett. 58 (1987) 1328; Phys. Rev. A 40 (1989) 5489; J. S. Nico, D. W. Gidley, A. Rich and P. W. Zitzewitz, Phys. Rev. Lett. 65 (1990) 1344.

[3] T. Mitsui, R. Fujimoto, Y. Ishisaki, Y .Ueda, Y. Yamazaki, S. Asai and S. Orito, Phys. Rev. Lett. 70 (1993) 2265; G. S. Atoyan, S. N. Gninenko, V. I. Razin and Y. V. Ryabov, Phys. Lett. B 220 (1989) 317.

[4] S. Asai, S. Orito, T. Sanuki, M. Yasuda and T. Yokoi, Phys. Rev. Lett. 66 (1991) 1298; D. W. Gidley, J. S. Nico and M. Skalsey, Phys. Rev. Lett. 66 (1991) 1302.

[5] K. Marko and A. Rich, Phys. Rev. Lett. 33 (1974) 980.

[6] T. Mitsui, K. Maki, N. Muramoto, T. Sato and S. Asai, in preparation.

[7] S. Asai, S. Orito, K. Yoshimura and T. Haga, Phys. Rev. Lett. 66 (1991) 2440; S. Orito, K. Yoshimura, T. Haga and M. Tsuchiaki, Phys. Rev. Lett. 63 (1989) 597.

[8] M. Tsuchiaki, S. Orito, T. Yoshida and M. Minowa, Phys. Lett. B 236 (1990) 81.

[9] M. V. Akopyan, G. S. Atoyan, S. N. Gninenko and V. V. Sukhov, Phys. Lett. B 272 (1991) 443.

[10] S. Asai, K. Shigekuni, T. Sanuki and S. Orito, Phys. Lett. B 323 (1994) 90.

[11] T. Chang, M. Xu, and X. Zeng, Phys. Lett. A 126 (1987) 189.

[12] T. Hyodo et al., in positron Annihilation, edited by L. Dorikens-Vanbraet, M. Dorikens and D. Segers (World Scientific, Singapore, 1989), p. 878.

[13] G. S. Adkins, Ann.Phys. (N.Y.) 146 (1983) 78. 


\section{Figure captions}

Fig. 1. A schematic view of the experimental setup. Circular inset: a magnified view of the source region.

Fig. 2. The time spectrum between the scintillator and germanium detector signals.

Fig. 3. The $o-P$ s spectra after subtracting the accidental contribution. (a) Below $160 \mathrm{keV}$. (b) Up to $600 \mathrm{keV}$.

Fig. 4. The resultant upper-limits at $90 \%$ C.L. on the branching ratio of $\gamma X^{0}$ decay in comparison with the existing limits [8]. 


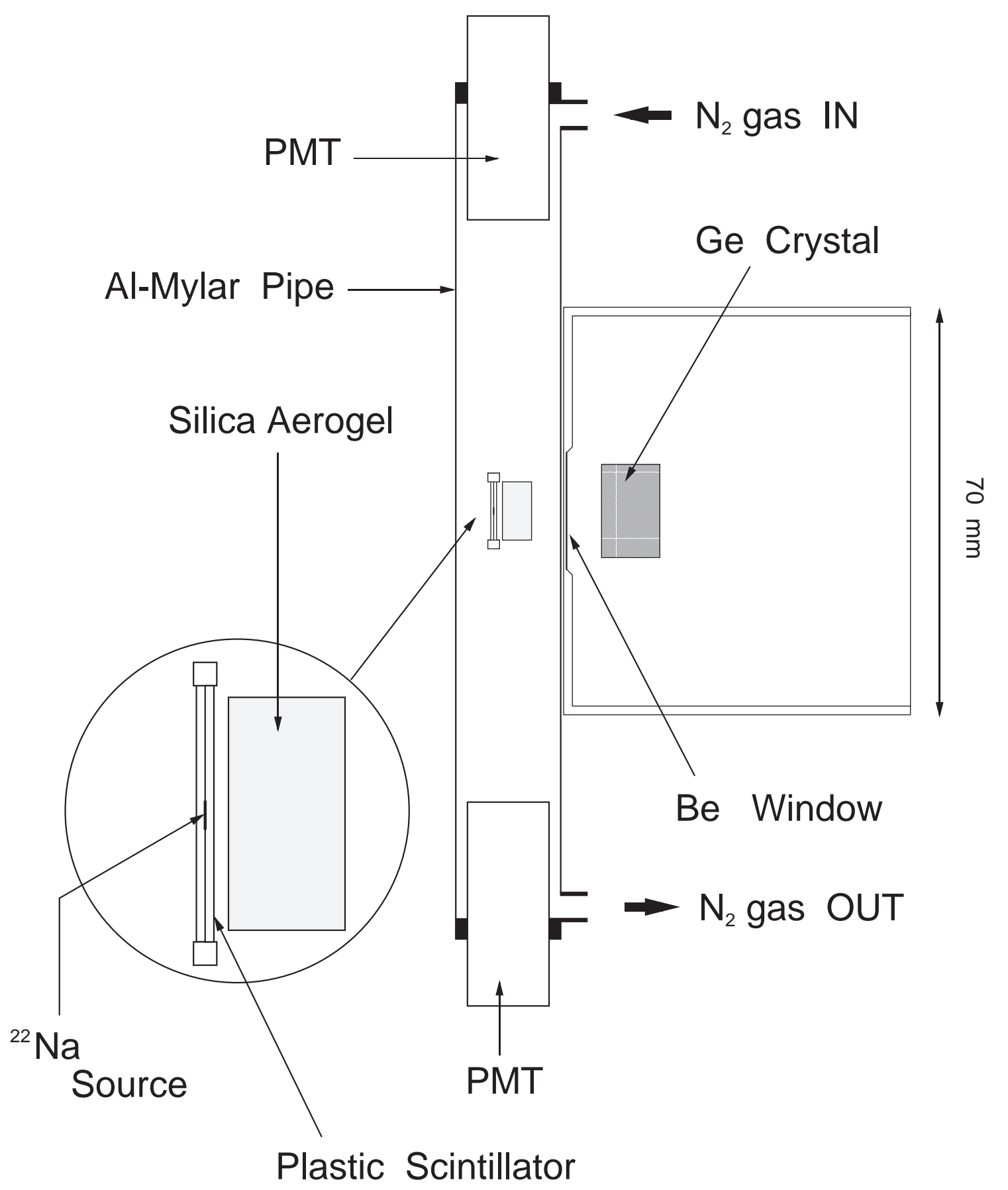

Fig. 1 


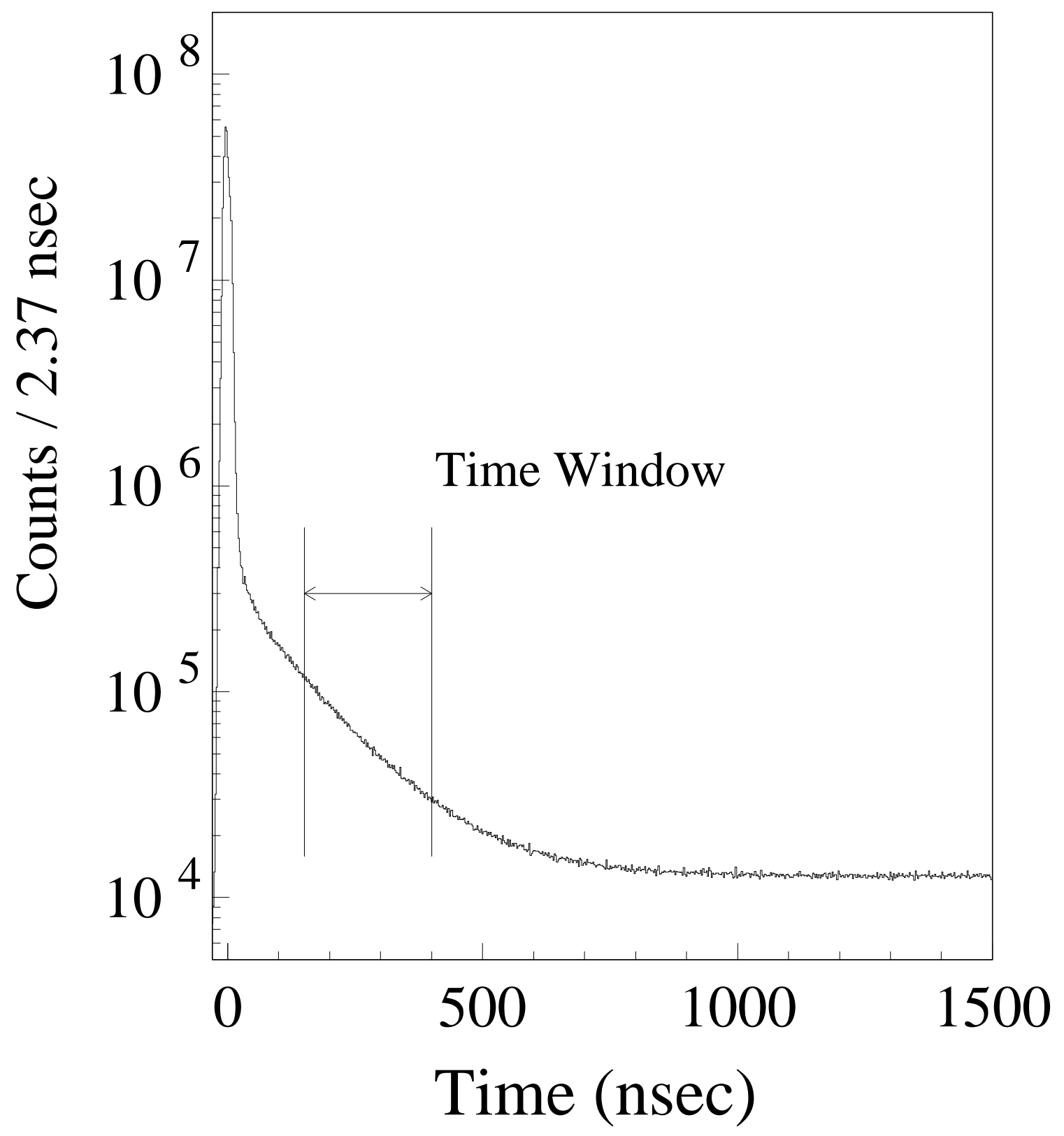

Fig. 2 

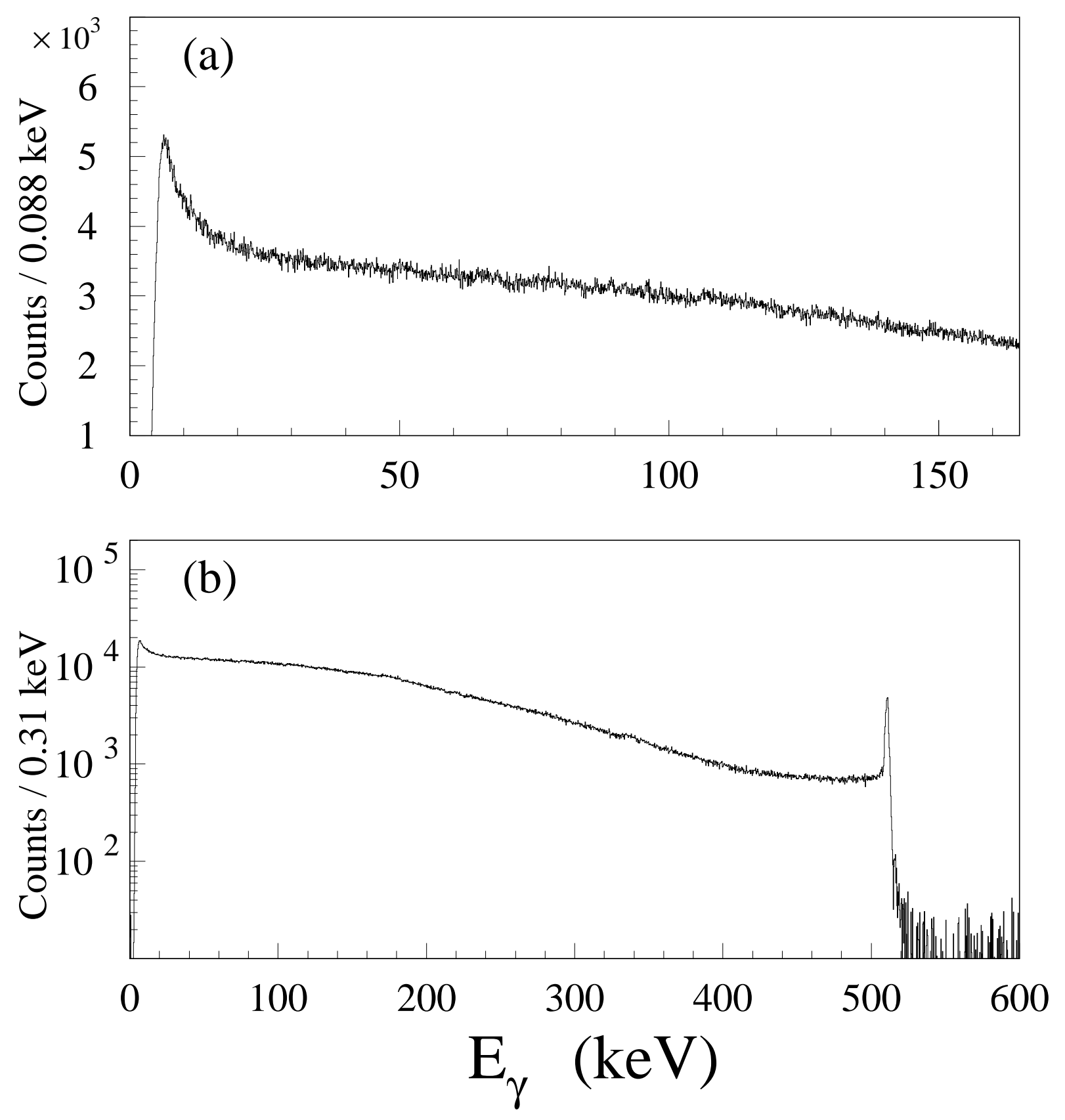

Fig. 3 


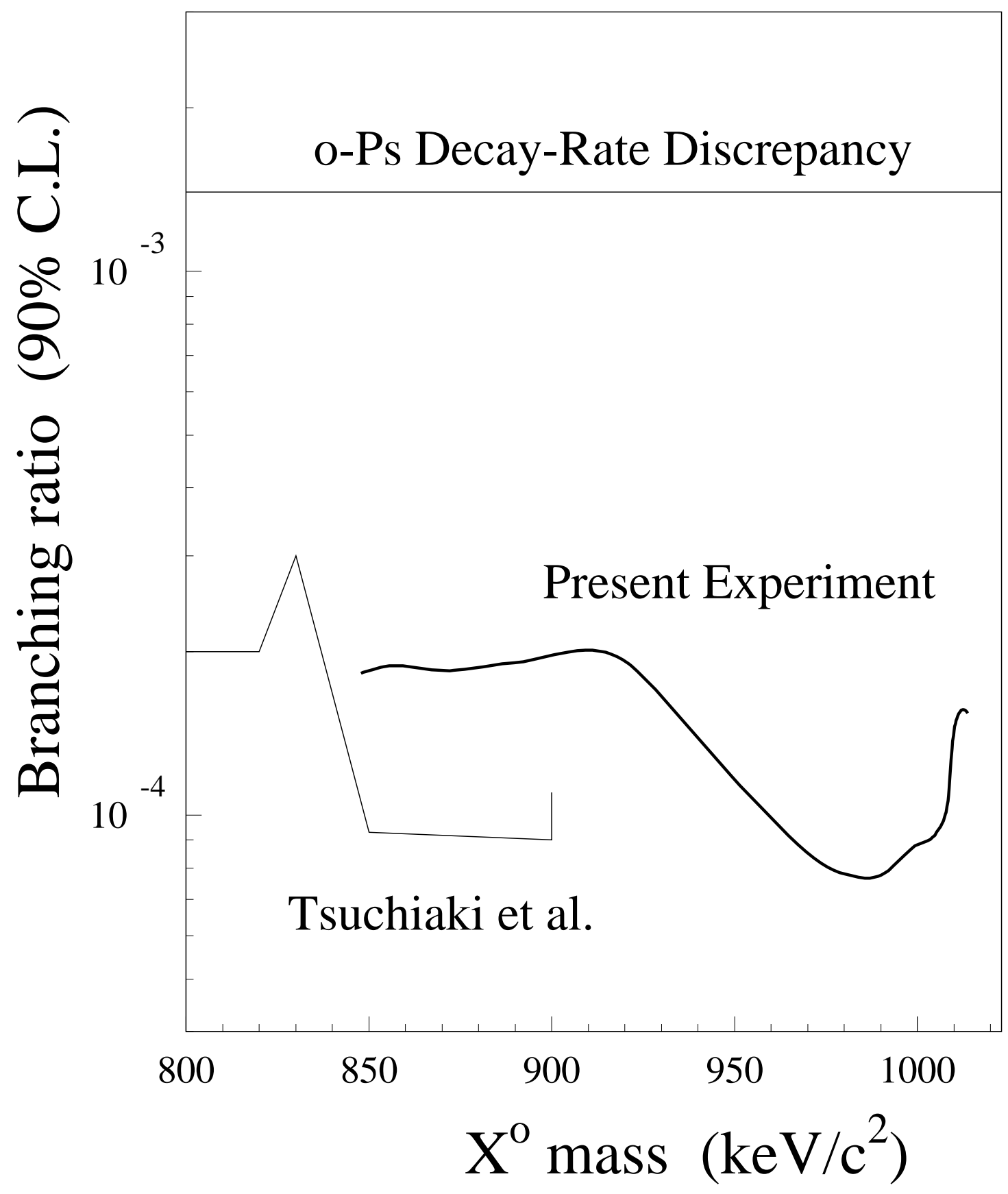

Fig. 4 